\title{
Testing Cooperative Communication Schemes in a Virtual Distributed Testbed of Wireless Networks \\ G. Kormentzas $^{1}$, L. Alonso ${ }^{2}$, and Ch. Verikoukis ${ }^{3}$ \\ gkorm@aegean.gr, luisg@tsc.upc.edu, cveri@cttc.es \\ ${ }^{1}$ University of the Aegean (AEG) \\ ${ }^{2}$ Technical University of Catalonia (UPC) \\ ${ }^{3}$ Telecommunications Technological Centre of Catalonia (CTTC)
}

\begin{abstract}
-
It is expected that Next Generation Networks (NGNs) will offer seamless interoperability among heterogeneous access technologies in order to provide ubiquitous access. In such settings, short range technologies may be used in order to extend the coverage area of cellular systems while cooperative diversity can improve the efficiency of the wireless systems. An advanced, backward compatible, with the 802.11 standard, MAC protocol for cooperative ARQ scenarios in NGNs sets the research framework for this work. The functionalities of the RCSMA protocol [1] will be enhanced and the derived analytical models will be validated at the UNITE Virtual Distributed Testbed (VDT) ([2]).
\end{abstract}

Keywords - ad hoc networks, medium access control protocols, cooperative communications, next generation networks, Cooperative ARQ.

\section{INTRODUCTION}

The network of the future seems to be a network of diverse wireless access technologies where the end-users will be able to attain any service, at any time, at the access that is optimised for the specific service resulting in a "flexibility and choice" that enhances the quality of life of the individuals. To this vision, the ubiquitous access in a costeffective way in an era where spectral resources are important, constitutes an emerging challenge.

Focusing on the network of the future, the Long Term Evolution (LTE) that evolves HSDPA is going to offer a communication framework with higher-data-rate, low-latency and packet-optimized radio-access technology for both uplink and downlink. The 3GPP System Architecture Evolution (SAE) encompasses an IP core network infrastructure with IP interfaces targeting multiple RATs (Radio Access Technologies), which will not only be restricted to 3GPP legacy systems such as UMTS Terrestrial Radio Access Networks (UTRAN) or GSM/EDGE Radio Access Network (GERAN), but will also have inherent open interfaces to accommodate wireless networks (WLAN, WMAN) through the IEEE 802.21 Media Independent Handover Function. In this context, the research of new transmission paradigms and techniques as the so-called cooperative communication schemes, have been getting special interest in the very last time.
The proposal herein presented addresses cooperative ad-hoc networking and considers heterogeneity with the final aim to ensure undiminished service perception whilst the subscriber terminal migrates freely between networks that might belong to different operators. We will consider enhancement techniques, which include networking to provide "virtually" collocated cellular and wireless coverage reflecting a converged network infrastructure. In this case the ad-hoc network, comprising ad hoc relay-capable nodes can be used to "fill" (guarantee the coverage of) the regions that are not covered by a cellular (WiMAX or UMTS-LTE) or an infrastructure-based WLAN. In such a kind of scenarios we identify the characteristics of a MAC protocol for cooperative Automatic Repeat reQuest (ARQ) and we define framework for a realistic performance evaluation.

The paper is organised as follows: Initially, the reference scenario of the EU funded project PASSENGER (Provision of optimum radio AcceSS at the Emerging Next GEneration NetwoRks) is presented as an indicative scenario of the network of the future. Then, a literature review of cooperative communications is presented. Section IV analyses the UNITE Virtual Distributed Testbed (VTD) that will be employed for the validation of the proposed cooperative communication schemes. The way how the variants of the advanced MAC protocol for cooperative ARQ and optimization mechanisms will be uploaded at the UNITE framework is discussed at Section V. Finally, Section VI concludes the paper.

\section{PASSENGER Scenario}

In the context of PASSENGER project an innovative scenario is proposed considering the joint application of session roaming and ad-hoc networking with relay-based connectivity. In this case the ad hoc network comprising relay-capable nodes can be used to "fill" the regions that are not covered by the WiMAX/UMTS-LTE or the infrastructure-based WLAN. This scenario requires the existence of an additional WR (Wireless Router) on the WLAN side which will keep connections with both the infrastructure-based and the ad hoc networks. For a node belonging to the ad hoc network the WiMAX/UMTS-LTE and the infrastructure-based WLAN networks are virtually collocated in the sense that the test node can use any of them to gain access to the "outside" world. Central entity in this scenario is a multi-radio wireless router (WR), equipped with 
both WLAN and WiMAX/UMTS-LTE network airinterfaces. This entity is not just a conventional node in a general ad hoc network but possesses enhanced functionality by being able to communicate with the WiMAX/UMTS-LTE network thus providing internet connectivity to the rest of the ad hoc nodes. Technical challenges in this scenario are, among others, the functionality of the WR, network discovery and association mechanism, the multi-hop routing protocol used in the ad-hoc network, and cooperative ARQ protocols.

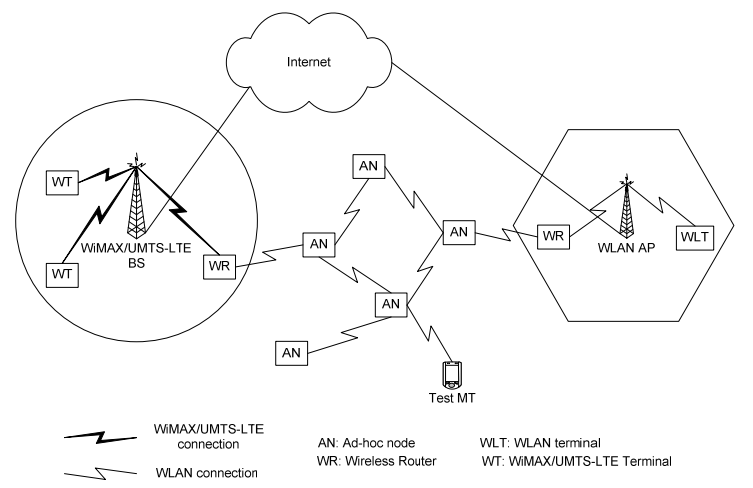

\section{Figure 1: Ad-hoc networking to provide "virtually" collocated WiMAX/UMTS-LTE and WLAN.}

In this work, we focus on the research framework of MAC protocols for cooperative ARQ schemes. Preliminary results of the Relay Carrier Sensing Multiple Access (RCSMA) [1] protocol in ARQ scenarios in wireless systems call for more extensive research in heterogeneous wireless networks. RCSMA makes an advance on the state of the art by introducing the petitioner concept in the operation of the protocols, where cooperation can be claimed by the receiver. In this work, the operation of the protocol is enhanced by introducing the persistency concept. In addition, the use of advanced Radio Resource Management techniques based on Cross-Layer algorithms in the selection of the adequate set of relays in order to guarantee certain QoS and energy saving mechanisms will further optimize the performance of the protocol. Likewise, the overhead introduced by the cooperation phase will be also considered as an advanced in the state of the art since all the proposed in the literature MAC protocols only focus on the cooperation process.

\section{State-of-the-art}

The main reasons for introducing ad hoc networks in existing wireless systems are to increase and improve the services for packet users, as well as to reduce the cell planning difficulties for operators. The potential of wireless ad hoc networks was investigated by several EU research projects. For example, the FleetNet project developed a wireless multi-hop ad hoc network for inter-vehicle communication to improve the driver's and passenger's safety and comfort. The ISTMobileMAN project investigated the potential of the Mobile ad hoc network paradigm. Specifically, the project aimed at the definition and development of a metropolitan area, self- organizing, and totally wireless network called Mobile Metropolitan Ad hoc Network (MobileMAN). In the project IST-ROMANTIK, multi-hop architectures for capacity and coverage enhancement for $3 G$ and beyond-3G wireless systems were investigated. Finally, exploitation of ad-hocnetworking aspects for coverage enhancement is currently being studied in the scope of the IST-WINNER project.

The broadcast nature of wireless communication systems makes possible to improve their performance by allowing users cooperating with each other. In multi-user environments, the use of distributed diversity seems to offer an interesting alternative to overcome the practical implementation drawbacks found out when experimenting with Multiple Input Multiple Output (MIMO) techniques using relatively small devices. The basis of cooperative communications is to exploit the fact that in the wireless channel, any transmission can be overheard by all the nodes within a certain transmission range; once a message is transmitted from a source to a given destination node, all nodes in the transmission range of the source station become potential helpers, referred to as relays, that could help out in the communication link. Since nodes in the network may be spatially distributed, the different copies received at each node can be used to create a spontaneous time and/or spatial diversity scheme. The improvement induced by exploiting cooperation in wireless networks can be achieved in terms of higher transmission rate, lower transmission delay, more efficient power consumption, or even increased coverage range.

The fundamental theory behind the concept of cooperation has been deeply studied among researchers during the last years [1]-[4] while significant effort and huge amount of innovative results for cooperation in the physical layer have been presented in the literature. However, significant less effort has been dedicated to the development of higher layer protocols.

Automatic repeat request (ARQ), which requests the data link layer of the transmitter to repeat the packet when a packet is erroneously received, had been widely used in wireless communications systems. Therefore, if multiple nodes had also received a copy of the transmitted, they could collaborate on the retransmission of such packet when needed and could increase the coverage area of a cellular network. This is known as Cooperative ARQ (C-ARQ) and introduces the cooperative transmission concept into the Date Link Control. C-ARQ topics have been addressed in several works in the literature. In [7], the SNR gain and average number of retransmissions of a single source cooperative ARQ protocol is studied. In [5] the saturation throughput of three doublesource cooperative ARQ protocols is presented. Cerutti and al. present in [6] a delay model for single-source and singlerelay cooperative ARQ protocols. They propose a simple set of retransmissions rules and their aim is to reduce the signalling and control overhead in the network, the hardware and algorithm complexity. In [8] three ARQ protocols are 
presented. In the first protocol the relay node always retransmits the packet. In the second, only the one with the better channel conditions between the relay and the transmitter is requested to repeat the packet. Likewise, spacetime codes are used in order to repeat simultaneously the packet transmission in the third protocol. Finally, in [9] Morillo et all propose a collaborative ARQ protocol that exploits diversity through collaboration in wireless networks. They demonstrate that when $\mathrm{M}$ neighbouring nodes collaborate using the proposed algorithm can get the same efficiency as an array of $\mathrm{M}$ antennas. In most of the previous works on cooperative transmission focus is put on analyzing the gains of cooperation from a fundamental point of view and simple TDMA schemes are considered. Therefore, the design of MAC protocols which coordinate the cooperative retransmissions in is an interesting research challenge.

Finally, some projects funded by the $6^{\text {th }}$ and $7^{\text {th }}$ framework programs include cooperative communications in their objectives. The aim of IST-COOPMAC Project is to provide the advances that will permit the deep understanding of both the theoretical and practical aspects of cooperative and opportunistic communications. The IST-FIREWORKS project aims to improve the current IEEE 802.16 by an enhanced multi-hop relaying deployment concept using cooperative technology. The ICT-CODIV will try to solve open problems on cooperative transmission both in the Physical and MAC layers while the ICT-REWIND will address cooperative relaying schemes in the framework of WIMAX-based systems.

Regarding distributed test-beds, several EU funded projects such as the IST-UNITE, the ICT-PII and the ICT-One Lab2 propose different solutions for the interconnection of federated testbeds for Future Internet Research and Experimentation.

\section{Overview of the UNITE framework}

The UNITE VDT system aims to address two main functional requirements. The first relates to the derivation of a simulation facility for composite wireless networks by interconnecting existing simulators of diverse radio technologies running on different platforms. The second has to do with the incorporation of facilities for joint Radio Resource Management functions (called URRM functions).

These requirements can be realized by the functional architecture depicted in Figure 2. This architecture relies on the identification of four conceptual levels for the distributed virtual testbed and the definition of discrete building blocks residing at these levels. At the topmost level, the end-user interface provides access to the UNITE controller, which is responsible for connecting the VDT platform with the external world. Using this interface and the facilities provided by the UNITE controller (e.g., identification of available simulators, retrieval of simulator parameters etc.), end-users compile simulation plans and submit them for execution (plans are created through a Scenario Editor, called VDT
Simulation Plan Editor). Additional functionalities of this component include management of user authentications and permissions, retrieval of past simulation plans from the repository, validation of simulation plans, scheduling of the simulation instance execution and storage of simulation plans to the repository for future use (the repository is implemented as part of the UNITE Data Base - UDB).

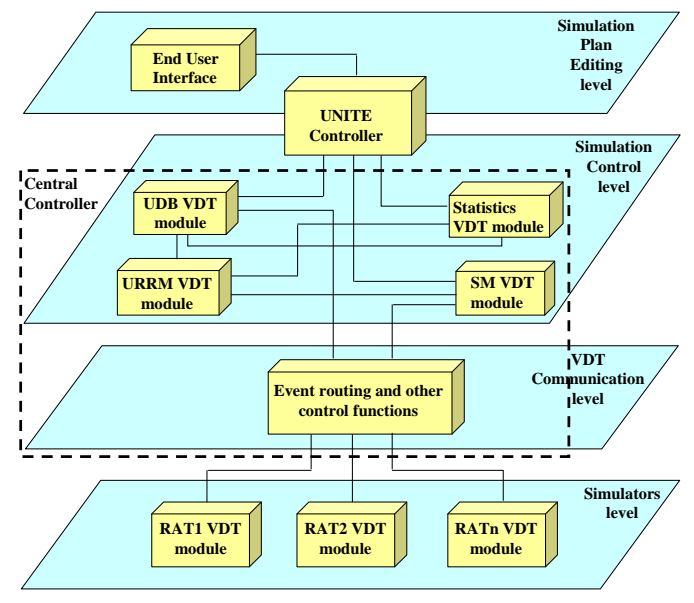

Figure 2: The UNITE VDT logical levels

When a simulation is eligible for execution, the UNITE controller hands over control to the Central Controller. The latter is comprised of four modules:

- the UDB module responsible for storing simulation results and simulation plans,

- the statistics module that performs statistical processing on the data of the UDB,

- the module that implements the joint URRM algorithm, the Scenario Manager (SM) that undertakes functions like terminal management, service and traffic stream management and simulator clusters time management.At the lowest level of the VDT architecture, there are a number of modules responsible for simulating radio technologies. Legacy simulators are attached to the distributed virtual platform by forming VDT modules. These modules are comprised of three entities (Figure 3):

- the actual simulator (in general, the federated service),

- the federated gateway which manages the simulation cluster and translates simulator-specific messages to VDT messages and vice versa,

- the VDT module API, which provides the interface (functions and parameter definitions), through which VDT modules communicate with the components of the Central Controller.

The Federated Gateway (FG) communicates with the other entities of the distributed testbed and transforms messages received from the testbed to simulator-related actions. The simulator calls specific functions from the Federated Gateway to send VDT Events. On the other hand, a specific function from the simulator is called each time a VDT Event is 
received from the VDT Central Controller, so that the event can be processed.

The communication framework is supported by an eventbased middleware implemented using SOAP over HTTP. The control of the events and their routing towards the distributed components registered for them is undertaken by the Central Controller. The whole translation of events (SOAP messages) to simulator-specific functions is implemented through an API, providing functions and parameter definitions, in order simulator developers to easily attach their simulation engine to the VDT.

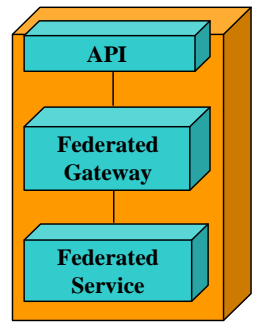

\section{Figure 3: The structure of a VDT module}

The VDT module is the basic building block of the VDT architecture and it can be used for any function that needs to be implemented in a separate hardware/software environment. In general, the VDT module can be considered as an abstract implementation of a desired function serving a specific purpose. In this regard, the notion of the VDT module can perfectly apply as well to the simulation control functions of the Central Controller (in fact, Figure 2 has been sketched with this assumption in mind). Additionally, the federated service of a VDT module can also correspond to an emulated or physical networking testbed.

\section{Research Methodology}

In PASSENGER the proposed research methodology includes analysis, using Markov chains, and validation, by means of simulation using the UNITE VDT, of the proposed protocols in different scenarios and conditions.

\section{Development of PASSENGER Algorithms}

Several infrastructure technologies such as WiMAX (802.16 $\mathrm{e} / \mathrm{j})$, UMTS-LTE, WLAN (802.11 b/g/n) will be taken into account in the proposed scenario while WLAN (802.11 b/g/n) will be considered for the ad hoc network.

The proposed MAC protocol will be based on the RCSMA protocol for cooperative ARQ. In RCSMA a new control packet was defined at the MAC layer in order to Claim For Cooperation (CFC). This CFC could be implemented following the structure of the RTS packet considered in 802.11-based systems, but indicating that both the source and destination of the data packet are the address of the node asking for cooperation. When a destination node needs to ask for cooperation, it must listen to the channel for a Short Inter Frame Space (SIFS) time. If the channel remains idle for that time, it will be able to broadcast the CFC. It is worth mentioning that the operation of the MAC protocol defined in the IEEE 802.11, sets a longer IFS (named DIFS) before getting access to the channel for the transmission of a data packet. Therefore, cooperation processes in RCSMA get higher priority than regular transmissions. At the reception of the CFC, the source node knows that has to wait for a cooperation procedure instead of executing the back-off procedure triggered by the lack of an ACK reception. On the other hand, the relay set initiates the cooperation process with the destination node. During the cooperation phase, the set of relays use the access rules defined in the 802.11 standard. Once the destination node is able to have a correct copy of the original packet, it is responsible for indicating to the source node and to its neighbouring nodes that the required cooperation procedure has finished. To do so, an ACK packet is transmitted.

In the context of PASSENGER, persistency is used, where the relays nodes continuously transmit the requested packet in order to overcome the problem of non sufficient number of relays [1]. It is worth mentioning, that the proposed protocol is backward compatible with the existing MAC protocols in 802.11 standards in order to be easily exploited in the near future. Analysis is based on Makov Chains and existing models such as the ones proposed by Bianchi and Wu have been adapted to evaluate the efficiency of the proposed protocol. Both saturated and non-saturated network conditions for the ad hoc nodes are considered. In the former case, all ad hoc nodes in the system have already a packet for transmission in their buffer and when cooperation is claimed they use the existing back-off window. In the later case, we consider that all nodes reset their back-off window and initiate a random back off period in order to avoid collision in the first transmission whenever cooperation is claimed.

The protocol will be further optimized by minimizing the overheads due to the cooperation phase. Moreover, we will address the relays selection problem by using advanced Radio Resource Management algorithms based on cross-layer techniques. Therefore, channel conditions, loss-rates, energy consumption, interference, traffic load and status of node's battery may be considered, among other variables, in the selection of the adequate set of relays in order to optimize the average contention time and/or the total energy consumption. Finally, the aforementioned protocol will be extended in order to cover various schemes of cooperative relaying and various options for relay nodes (decode and forward, amplify and forward etc...). The previous scenarios will be studied both with fixed and mobile relays. Depending on the scenario and the application the previous schemes will be studied under QoS and energy constraints.

The proposed protocol will be evaluated in terms of the required delay to recuperate the original packet, the total throughput of the system and the total energy consumption, for different values of the back-off window and number of cooperative relay nodes. 


\section{Validation of PASSENGER Algorithms}

PASSENGER algorithms are intended to run on separate URRM modules that will be VDT modules attached to the VDT system. At this case, the Federated Service of these VDT modules will not be simulators but the algorithms themselves. The following issues have to be addressed:

Implementation of the URRM

- Implementation of the URRM VDT modules

- Attaching of the URRM VDT modules to the central controller

\section{Storing of simulation results}

- Definition of results to be stored into the UDB

- Definition of the structure of these results when sent through the UpdateValues event

- Implementation of storing of these results to the UDB

- Testing

\section{Retrieving of simulation results}

URRM must retrieve simulation results from the UDB in order to take decisions. This is done through the getSystemInformation or getMobileInformation events. The following have to be addressed:

- Definition of the parameters that each event will have

- Definition of the structure of the information that each event will contain. For example, getMobileInformation will contain all the simulation results concerning a specific mobile. How will these results be extracted from the event in the Federated Gateway of the URRM VDT?

- Implementation of retrieving of the results from the UDB, packing them in a predefined structure and embodying them in the getSystemInformation or getMobileInformation events, when requested.

- Testing

\section{Service IDs}

Three services are supported so far with static parameters. Service parameters must be dynamic:

- Definition of more parameters in the SendStreamToMobile and StartTxToMobile events, so as to support dynamic configuration of their parameters, which will be dependent to each service

- Change in the definition of the scenario story so as to support dynamic configuration of service parameters

- Implementation of the new scenario story to the VDT editor

- Implementation of reading of the new scenario story from the UNITE controller and embodying the service ID parameters in the SendStreamToMobile and StartTxToMobile events.

- Testing

\section{QoS parameters}

Each service ID should be accompanied with QoS parameters, the values of which should be able to be configured dynamically by the user:
- The steps for the case of service IDs stand in this case as well.

After all the above issues are addressed, the steps needed to integrate the algorithm are the following:

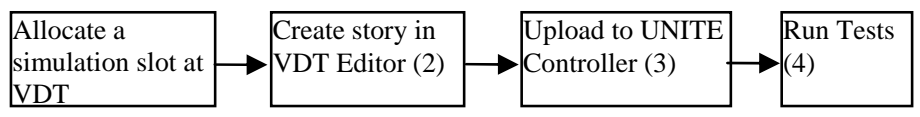

\section{Conclusions}

The UNITE Virtual Distributed Testbed constitutes an ideal environment where cooperative communications schemes could be validated. Building at this potential, the paper discusses how the PASSENGER schemes are going to be uploaded and tested at VDT. In this context a synergy between two EC funded projects is achieved.

\section{REFERENCES}

[1] J.Gomez, J.Alonso, Ch.Verikoukis, A. Pérez-Neira and L.Alonso, "Cooperation on Demand Protocols for Wireless Networks”, IEEE PIMRC2007, Athens, September 2007.

[2] D.Vassis, L.Sarakis, G.Kormentzas and Ch.Verikoukis, “A Distributed Testbed for Performance Evaluation of Inter-system Optimization Schemes in Heterogeneous Wireless Networks", in the Middleware Technologies for Enabling Next-Generation Network Services and Applications multicon lectures notes published by Multicon Verlag. ISBN 978-3-930736-12-6

[3] T. M. Cover and A. E. Gamal, "Capacity Theorems for the relay channel”, IEEE Transactions on Information Theory, vol. 25, no. 5, p. 572, Sept 1979.

[4] A. Sendonaris, E. Erkip and B. Aazhang , "User cooperation diversity-part I: System Description”, IEEE Transactions on Communications, vol. 51, no. 11, pp. 1927-1938, Nov. 2003.

[5] A. Sendonaris, E. Erkip and B. Aazhang, "User cooperation diversity-part II: Implementation aspects and performance analysis, "IEEE Transactions on Communications, vol. 51, no. 11, pp. 1939-1948, Nov. 2003.

[6] P.Gupta, I.Cerruti and A. Fumagalli, "Three transmission scheduling policies for a cooperative ARQ protocol in radio networks”, In Proc. WNGG conference, October 2004.

[7] I.Cerruti, P.Gupta and A. Fumagalli, "Delay Model of a OneWay Cooperative ARQ Protocol in Slotted Radio Networks with Poisson Frames Arrivals" IEEE Transactions on Networking, Jan 2007.

[8] E. Zimmermann, P. Herhold, and G. Fettweis, "The impact of cooperation on diversity-exploting protocols," in Proc. Of $59^{\text {th }}$ IEEE Vehicular Technology Conference 2004.

[9] C. Yu, Z. Zhang and P. Qiu, "Cooperative ARQ in Wireless Networks: Protocolos Description and Performance Analysis”, IEEE ICC 2005.

[10] J. Morillo-Pozo, J. García-Vidal, A.I. Pérez-Neira, "Collaborative ARQ in Wireless Energy-Constrained Networks", Proceedings of the workshop on Foundations of Mobile Computing 2005 (DIAL-POM’05). 